\title{
O IMPACTO ECONÔMICO DO TRAUMA EM UM HOSPITAL UNIVERSITÁRIO
}

\author{
ECONOMIC IMPACT OF TRAUMA IN AN UNIVERSITY HOSPITAL
}

\author{
Luiz Carlos von Bahten, TCBC - PR ${ }^{1}$ \\ Emanuelle de Mello Alcantara ${ }^{2}$ \\ Ana Paula Piai Pimenta ${ }^{3}$ \\ Juliana Corrêa Dallagnol ${ }^{3}$ \\ Kelly Okamoto Yoshizumi ${ }^{3}$ \\ Marianna Ferreira Dresch ${ }^{3}$
}

\begin{abstract}
RESUMO: Objetivos: Determinar o impacto financeiro do trauma em um hospital universitário em Curitiba. Método: Estudo retrospectivo de pacientes vítimas de trauma, internados no Hospital Universitário Cajuru, divididos de acordo com o mecanismo de trauma. Foram analisados o número de dias de internamento e o custo assistencial total de cada paciente declarados na ficha de Autorização de Internação Hospitalar (AIH). A partir daí, calculou-se o custo médio, a média de dias de internamento e o custo médio paciente/dia. Resultados: O custo médio de 349 pacientes estudados foi de US\$ 568,22; das vítimas por ferimento por arma de fogo (FAF), US\$ 692,50; das vítimas de ferimento de arma branca (FAB), US\$ 676,9; das vítimas de agressão, US\$ 412,3; das vítimas de queda, US\$ 503,05 e das vítimas de acidente de trânsito, US\$600,30. A média de dias de internamento da amostra total foi de 6,2; das vítimas de FAF, 7,7; das vítimas de FAB, 5,3; das vítimas de agressão, 6,7; das vítimas de queda, 7 e das vítimas de acidente de trânsito 6. O custo médio paciente/dia da amostra total foi de US\$ 91,65; das vítimas de FAF, US\$ 89,99; das vítimas de FAB, US\$ 127,66; das vítimas de agressão, US\$ 61,54; das vítimas de queda, US\$ 71,86 e das vítimas de acidente de trânsito, US\$ 100,05. Conclusão: Este estudo, como tantos outros, mostra que o trauma é uma doença negligenciada, importante para a saúde publica por seu grande impacto social e econômico e passível de redução através de medidas de prevenção.
\end{abstract}

Descritores: Ferimentos e lesões; Gastos com hospitalização; Fatores socioeconômicos; Prevenção; Hospitais universitários.

\section{INTRODUÇÃO}

O trauma foi cognominado como a "doença negligenciada da sociedade moderna" porque mata e incapacita mais do que as guerras, mas não sensibiliza nem mobiliza a sociedade e os governos. ${ }^{1}$ É a principal causa de morte nas quatro primeiras décadas de vida e é responsável pela perda de mais anos de vida produtiva do que a doença cardíaca e o câncer combinados. ${ }^{2,3}$

O prejuízo social que o trauma traz é imensurável. Não há como avaliar quantitativamente o impacto social que as mortes ou seqüelas do trauma causam para a população. É possível, porém, fazer

1. Professor Adjunto das disciplinas de Morfologia e Atendimento Inicial ao Traumatizado pela Pontifícia Universidade Católica do Paraná; Professor Adjunto da disciplina do Trauma da Universidade Federal do Paraná; Mestre em Cirurgia pela Universidade Federal do Paraná e Doutor em Cirurgia pela Universidade de Campinas.

2. Farmacêutica pela Universidade Tuiuti do Paraná e acadêmica do $5^{\circ}$ período de medicina da Pontifícia Universidade Católica do Paraná.

3. Acadêmicas do $5^{\circ}$ período do curso de medicina da Pontifícia Universidade Católica do Paraná.

Recebido em: 02/12/2002

Aceito para publicação em: 17/04/2003

Trabalho realizado no Hospital Universitário Cajuru - Pontifícia Universidade Católica do Paraná. 
uma avaliação do impacto econômico que essas vítimas trazem para a sociedade. Através de cálculos estatístico-epidemiológicos, é possível estimar qual o gasto financeiro que um traumatizado representa, considerando-se seu atendimento, acompanhamento, reabilitação ou perda de capacidade produtiva.

A este gasto se juntam os demais prejuízos e danos de ordem material e social ocasionados ao meio ambiente e às máquinas, equipamentos, vias e próprias comunidades onde ocorrem os eventos. Por outro lado, são necessários técnicas e recursos humanos altamente qualificados, para tornar viável a implementação das medidas de prevenção e promoção de segurança em âmbito local e global. ${ }^{1}$

Em um estudo com 250 pacientes, vítimas de ferimentos por arma de fogo, Wintemute e Wright observaram que o custo total de tratamento e reabilitação de cada uma dessas vítimas foi de aproximadamente US\$14.982. ${ }^{4}$

Em outro estudo com 211 pacientes vítimas de ferimentos por arma branca ou arma de fogo, Clancy, Misick e Covington observaram que o custo de tratamento desses pacientes foi de US\$ 510.432,00 em um ano. ${ }^{5}$

Max, Stark e Root observaram que, após a implementação da lei que obriga o uso de capacetes para todos os motociclistas na Califórnia, houve uma redução nos custos do tratamento das lesões por acidentes de moto. Este estudo estima que três quartos dessa redução, tenham acontecido por diminuição dos custos de tratamento de traumas em cabeça ${ }^{6}$.

Ainda que o trauma não representasse um gasto econômico significativo, é de fundamental importância social já que reflete como a sociedade vem se comportando. A incidência do trauma vem aumentando em proporção direta ao crescimento da população ao número de veículos e à exposição ao risco. Entretanto, a exposição ao risco pode não depender apenas do aumento da população e do número de veículos.

O trânsito, no Brasil, mata oito vezes mais do que nos Estados Unidos, cinco vezes mais do que no Japão e na Inglaterra, três vezes mais do que na França e Alemanha, mostrando que a exposição ao risco obedece a um terceiro fator que não aquele determinado pelo aumento de população e de veículos. ${ }^{7}$

A exposição ao risco está firmemente ligada ao comportamento, ao grau de prudência, à maneira de agir e à maneira de sentir, que formam a maneira de se conduzir. Frente à grande dificuldade em se intervir no aumento da população e no crescimento da frota de veículos, propõe-se uma intervenção na exposição ao risco, ou seja, uma proposta de educar e prevenir a população. Estudos atuais mostram que um quarto das mortes por trauma podem ser prevenidas com medidas de educação e melhorias de cuidados.

Do ponto de vista da prevenção, propõem-se medidas de caráter social e situacional. As primeiras se referem à possibilidade de se criar uma sociedade não violenta e se constituem em medidas coletivas visando à equiidade, integração social, desarmamento, desenvolvimento urbano, prevenção do crime, antiterrorismo e políticas de antiviolência policial. A prevenção situacional se refere ao contexto imediato de um processo de violência determinado e tem como objetivo diminuir os riscos da manifestação explícita de violência, ou seja, do ato violento em si.

A proposta deste trabalho é de determinar, através de um estudo retrospectivo, o impacto financeiro do trauma em um hospital referência na cidade de Curitiba e, ainda, analisar, através da revisão de literatura, a prevenção como uma medida eficaz na redução desses custos.

\section{MÉTODO}

O projeto do presente estudo foi submetido à analise pelo Comitê de Ética de Pesquisa da Pontifícia Universidade Católica do Paraná, tendo sido aprovado para desenvolvimento por estar de acordo com a resolução 196/96.

Foi feito um estudo retrospectivo dos prontuários de todos os pacientes trazidos pelo SIATE (Serviço Integrado de Atendimento ao Trauma em Emergência) e internados pelo Serviço de Emergências do Hospital Universitário Cajuru (HUC) no período de setembro de 2000 a fevereiro de 2001, num total de 516 prontuários.

Dos 516 prontuários consultados foram considerados, para fins de estudo, somente aqueles que apresentavam dados completos de admissão, internamento, recursos financeiros e alta hospitalar, o que totalizou 349 prontuários. Estes 349 pacientes foram divididos em grupos, de acordo com o mecanismo de trauma, da seguinte maneira: 44 pacientes internados por ferimentos por arma de fogo (FAF), 24 por ferimentos por arma branca (FAB), 23 por agressão, 73 por queda e 185 por acidente de trânsito. O número de pacientes incluídos em cada grupo não representa uma amostra proporcional dentre 
os prontuários consultados devido ao critério de seleção utilizado neste trabalho.

Em cada prontuário consultado verificou-se o número de dias de internamento e o custo assistencial total de cada paciente, ambos declarados na ficha de AIH (Autorização de Internação Hospitalar) que é anexada ao prontuário de cada paciente após a alta. A AIH leva em consideração, para determinar os gastos: material utilizado no pronto-socorro, salas de cirurgia e material utilizado para o ato cirúrgico, diárias e material utilizado na UTI, diárias e material utilizado na enfermaria, medicamentos utilizados no pronto socorro, no centro cirúrgico, na UTI e na enfermaria, nutrição enteral e parenteral, exames laboratoriais e de imagem, sangue e hemoderivados e serviços profissionais.

Os valores foram obtidos em reais e corrigidos pela cotação média do dólar no período ( $\mathrm{R} \$ 1,8851)$. O tempo de internamento foi calculado a partir da data de admissão e data de alta do paciente, que são declaradas, juntamente com os custos totais, na ficha de AIH (Autorização de Internação Hospitalar).

De posse desses dados calculou-se, através de média aritmética simples, o custo médio de cada paciente e a média de dias de internamento por mecanismo de trauma. A partir disso, foi possível, ainda, calcular a média de custo paciente/dia por mecanismo de trauma, através da razão entre o custo médio e a média de dias de internamento.

\section{RESULTADOS}

O custo médio dos 349 pacientes, objeto deste estudo, foi de US\$ 568,22 e a média de dias de internamento foi de 6,2 . O paciente que representou maior custo para o hospital, teve um gasto de US $\$ 5.721,43$ e o que teve o menor custo, gastou US\$21,42. O maior tempo de internamento, entre estes pacientes, foi de 57 dias e o menor tempo foi de um dia. O custo médio por paciente por dia, em toda a amostra, foi de US\$ 91,65.

Para as 44 vítimas de ferimentos por arma de fogo analisadas, encontrou-se um custo médio de US\$ 692,95 e uma média de 7,7 dias de internamento, resultando, assim, em um custo médio de US\$ 89,99 por paciente/dia. O maior gasto, nessa amostra, foi de US\$4471,35 e o menor gasto foi de US\$21,42. O maior tempo de internamento foi de 31 dias e o menor tempo foi de um dia.

Os 24 pacientes vítimas de ferimentos por arma branca apresentaram custo médio de US\$676,59, sendo que o maior custo foi de US\$2139,42 e o menor foi de US\$ 42,85. A média de dias de internamento foi de 5,3 e o custo médio foi de US\$127,66 por paciente/dia. O paciente que ficou o maior número de dias internado permaneceu no hospital por 25 dias e o que ficou menos tempo internado permaneceu no hospital por apenas um dia.

Entre os 23 pacientes que sofreram agressão, a média de dias de internamento foi de 6,7 e a média de custos foi US $\$ 412,33$. O custo médio por paciente/ dia, obtido a partir desses dados, foi de US\$ 61,54. Entre essas vítimas, o maior gasto foi US\$1051,72 e o maior número de dias de internamento foi 17 dias. O menor gasto foi de US\$21,42 e o menor número de dias de internamento foi um dia.

Do total de 73 pacientes da amostra de vítimas por queda, a média de custos foi de US\$503,05 e a média de dias de internamento foi de sete, o que leva a um custo de US $\$ 71,86$ por paciente/dia. O paciente que representou maior gasto para o hospital, custou US\$ 5.721,42 e o que representou menor gasto, custou US\$ 21,42. Nessa amostra, o maior tempo de internamento foi de 39 dias e o menor tempo foi de um dia.

Para os 185 pacientes vítimas de acidentes de trânsito, encontrou-se uma média de seis dias de internamento, sendo que o maior número de dias foi de 57 e o menor, de um dia. O custo médio foi de US\$ 600,30 e a média por paciente/dia foi de US\$ 100,05. O valor mais alto gasto com essa vítimas foi de US\$ 2.722,02 e o mais baixo de US\$ 21,42.

\section{DISCUSSÃO}

Os valores apresentados neste trabalho podem não representar o custo real do tratamento dos politraumatizados admitidos pelo Serviço de Emergências do Hospital Universitário Cajuru porque foram obtidos a partir da $\mathrm{AIH}$, na qual é declarado o valor que o hospital recebe do Sistema Único de Saúde pelos custos de tratamento de cada paciente.

Ainda assim, pode-se perceber que os custos do tratamento das lesões traumáticas são significativos. Neste estudo, a hospitalização dos 349 politraumatizados, no período de setembro de 2000 a fevereiro de 2001, representou um custo de US\$203.989,94 para o Hospital Universitário Cajuru.

Deste total, o tratamento dos ferimentos por arma de fogo representa $14,9 \%$; dos feridos por arma branca, $8 \%$; dos que sofreram queda, 18\%; das 
vítimas de agressão, 4,7\% e dos acidentados no trânsito, $54,4 \%$

Estimou-se, ainda, que um politraumatizado fica, em média, 6,2 dias internado e que, para o hospital, o custo total de seu tratamento é de aproximadamente US $\$ 568,22$, representando um gasto de US\$ 91,65 por dia de internamento.

A análise da amostra, quando dividida em grupos de acordo com o mecanismo de trauma, mostrou que os pacientes vítimas de FAF tem uma maior média de dias de internamento $(7,7)$ e um custo médio (US\$692,95) maior que os pacientes internados pelos demais mecanismos de trauma. As vítimas de agressão são aquelas que representam o menor gasto: US\$ 412,33 por paciente, em média; porém, não são as que representam o menor número de dias de internamento.

O menor número de dias de internamento para uma vítima de trauma é o dos pacientes que sofrem FAB (5,3 dias, em média) e coincide com o maior custo por paciente por dia de internamento, que é de US\$127,66.

O menor custo por dia de internamento é de US\$ 61,54 e se verifica entre as vítimas de agressão que, apesar de serem as que têm a segunda maior média de tempo de internamento, são as que têm o menor custo médio.

A análise destes dados mostra que o custo de tratamento de um paciente politraumatizado é considerável. Existe, além das despesas com atendimento médico, um custo social enorme, representado por perdas de produção, de salários das vítimas, funerais, reabilitação dos feridos, despesas com órgãos públicos, com advogados, com recuperação dos veículo, das vias, além de outros gastos materiais.

Para o Estado, existe, ainda, a perda de investimento em educação, treinamento e especialização, quando a vítima de trauma sofre a lesão em plena fase produtiva, o que é bastante comum, já que o trauma é a principal causa de morte nas quatro primeiras décadas de vida.

Os acidentes de trânsito têm grande impacto no Brasil. O Departamento Nacional de Estradas de Rodagem (DNER), através da sua divisão de Engenharia, realizou estudos em que estima um custo médio por pessoa acidentada de US\$13.360,00. Na composição desses custos foram consideradas as despesas médicas, os danos materiais (veículos e vias) e a perda de rendimento desse acidentado durante o período de inatividade. Em 1990, foram registrados em torno de 320 mil vítimas (fatais e não fatais). Segundo padrões do DNER, estes acidentes representam um custo social na ordem de 4,3 bilhões de dólares. ${ }^{8}$

Uma série de estudos relacionando acidente de trânsito e gastos financeiros, demonstraram que estes gastos são reduzidos significativamente com medidas de prevenção. Assim, durante os primeiros dois anos após a implementação da lei do uso obrigatório de capacete para motociclistas, na Califórnia, houve redução dos custos das lesões e fatalidades, além de sobra de fundos para o Estado, comparado com anos anteriores. Tanto os custos diretos como os indiretos apresentaram decréscimo durante o primeiro ano em que esta lei esteve em vigor, e esta redução foi mantida durante o segundo ano. Os custos totais com hospitalização de motociclistas apresentaram redução de US\$ 79,5 milhões em 1991, para US\$ 50,9 milhões em $1992 \mathrm{e}$ para US\$ 51,4 milhões em 1993. Aproximadamente três quartos desta redução $(73 \%)$ foram atribuídos à redução de custos com tratamento de pacientes com lesões de cabeça. Houve, ainda, significativa diminuição no tempo de internamento destes pacientes, de 7,9 dias em 1991, para 7,3 e 6,6 em 1992 e 1993, respectivamente. ${ }^{6}$

Um outro estudo relacionou o consumo de álcool com o uso de capacetes por ciclistas. Os pacientes que consomem álcool são mais gravemente feridos e, portanto, geram maior custo. Esta pesquisa revela que os consumidores de álcool têm uma elevada taxa de morbidade e fazem parte de um subgrupo de vítimas de trauma que aumenta significativamente os gastos com saúde e que, certamente, se beneficiariam com programas de prevenção de acidentes. ${ }^{9}$

O presente estudo demonstrou que, apesar de apresentar menor incidência que os acidentes de trânsito, as lesões por arma de fogo são as mais custosas em termos de tratamento. Dentre os mecanismos de trauma elencados neste trabalho, os ferimentos por arma de fogo são os que apresentam maior custo médio e maior média de dias de internamento.

Ainda que os custos de tratamento desse tipo de lesão não sejam significativos, a alta média de dias de internamento representa um problema sério, já que a falta de leitos é um problema comum nos hospitais públicos brasileiros. 
Considerando-se que o trauma representa, para um hospital, um custo elevado é de extrema importância que se encontrem meios de reduzir ao máximo estes custos. Os hospitais, por si só, não têm condições de controlar os custos dos traumas. Portanto, medidas de prevenção primária e secundária são, certamente, a forma de controle de custos mais eficaz. ${ }^{10}$

Um princípio básico de medicina preventiva e saúde pública é que a remoção de um fator ambiental causador de lesão ou doença é, geralmente, mais efetivo, em termos de prevenção, que os esforços necessários para uma mudança de comportamento por parte da população. Para as lesões por arma de fogo, o fator ambiental em questão é o acesso às próprias armas de fogo. A melhor estratégia para o controle do acesso às armas de fogo é uma legislação de controle de armas. ${ }^{11}$
Como solução para os altos custos dos traumas penetrantes relacionados com a violência, serão necessárias atenção sociolegal e medidas educacionais voltadas diretamente às fontes primárias de violência. ${ }^{5}$

A melhora no atendimento pré-hospitalar e hospitalar é a única estratégia razoável para a redução da morbidade e da mortalidade das lesões por arma de fogo, caso elas já tenham ocorrido. Porém, quando se procuram métodos de reduzir os custos, referentes à mortalidade e à morbidade dos ferimentos por arma de fogo antes que eles aconteçam, somente a prevenção primária pode ser uma medida eficaz. ${ }^{11}$

A etiologia do trauma está na sociedade e nela a solução. Cada ação política, preventiva, terapêutica e de consciência debruçada sobre a pessoa humana é uma esperança para atenuar este flagelo. ${ }^{1}$

\begin{abstract}
Background: The aim of this study is to determine the economic impact of trauma in an university hospital in Curitiba. Methods: A retrospective study of medical registries was carried out and they were divided in accordance to trauma mechanism, number of in-hospital days and total medical assistance costs were also considered for each patient on the In-Hospital Authorization Form. From these data, the average cost, average in-hospital days and average patient/day costs were calculated. Results: The average cost for the 349 patients was US\$ 568.22; for fire gun victims, US\$ 692.50; for blade victims, US\$ 676.90; aggression victims, US\$ 412.30; fall victims and traffic accident victims, US\$600.03. The average in-hospital days for the total sample was 6.2; fire gun victims, 7.7; blade victims, 5.3; aggression victims, 6.7; fall victims, 7 and traffic accident victims was of 6 . The average patient/day cost, for the total sample, was US\$ 91.65; fire gun victims, US\$ 89.99; blade victims, US\$127.66; aggression victims, US\$ 61.54; fall victims, US\$71.86 and traffic accident victims, US\$100.05. Conclusions: This study corroborates with many others that show that trauma is a neglected disease, important to public health due to its enormous social and economic wich can be avoided by means of preventive measures.
\end{abstract}

Key Words: Wound and injuries; Socioeconomic factors; Expenditures, Hospital Preventive measures; Hospitals, university

\section{REFERÊNCIAS}

1. Batista JN, Gomes EGA - "Etiologia do trauma". In Freire E - Trauma: a doença dos séculos. $1^{\mathrm{a}}$ Edição. São Paulo: Atheneu, 2001. v. 1, pp. 17-45.

2. Biffl WL, Moore EE - «Trauma Múltiplo». In: Harken HA, Moore EE - Segredos em cirurgia. $3^{\text {a }}$ Edição. Porto Alegre: Artmed, 1999, p 93.

3. Jacobs BB, Jacobs LM - «Epidemiology of trauma». In Feliciano DV, Moore EE, Mattox KL - Trauma. $3^{\text {a }}$ Edição. Stamford: Appleton and Lange, 1996, pp.1539.
4. Wintemute GJ, Wright MA - Initial an subsequent hospital costs of firearm injuries. J Trauma. 1992, 33(4): 556-560.

5. Clancy TV, Misick LN, Covington D, et al. - The financial impact of intencional violence on community hospitals. J Trauma. 1994, 37(1): 1-4.

6. Max W, Stark B, Root S - Putting a lid on injury costs: the economic impact of the California motorcycle helmet law. J Trauma. 1998, 45(3): 550-556.

7. Queiroz JS - "Histórico do trauma". In Freire E - Trauma: a doença dos séculos. $1^{\mathrm{a}}$ Edição. São Paulo: Atheneu, 2001, v. 1, p. 3-15. 
8. Ribeiro L, Lordello J. Como conviver com a violência. $1^{a}$ Edição. São Paulo: Moderna, 1998, pp. 189-194.

9. Spaite DW, Criss, EA, Weist DJ, et al. - A prospective investigation of the impact of alcohol consumption on helmet use, injury severity, medical resource utilization, and health care costs in bicycle-related trauma. J Trauma. 1995, 38(2): 287290.

10. Ordog GJ, Wasserberger J, Ackroyd G - Hospital costs of firearm injuries. J Trauma. 1995, 38(2): 291298.
11. Mock C, Pilcher S, Maier R - Comparison of the costs of acute treatment for gunshot and stab wounds: further evidence of the need of firearms control. J Trauma. 1994, 36(4): 516-521.

Endereço para correspondência:

Rua Silveira Peixoto n ${ }^{\circ} 780$ apartamento 501.

Bairro: Batel - CEP: 80240-120 Curitiba-PR

Telefones: (041) 242-3704 / 343-0474 / 9901-4393

E-mail:jucd@netpar.com.br 